\title{
Experimental results for active control of multimodal vibra- tions by optimally placed piezoelectric actuators
}

\author{
Andrea Rossi ${ }^{1, *}$, Fabio Botta ${ }^{1}$, Roberto Maiozzi ${ }^{1}$, Andrea Scorza ${ }^{1}$, and Salvatore Andrea \\ Sciuto ${ }^{1}$ \\ ${ }^{1}$ Dipartimento di Ingegneria, Università degli Studi Roma Tre, Via della Vasca Navale, 79, 00146 Rome, \\ Italy.
}

\begin{abstract}
Vibration damping is an effective strategy to enhance the life-cycle and performance of mechanical components. In this regard passive control systems involve lower costs and are easier to implement but their bandwidth is limited, whereas active systems provide larger bandwidth and higher adaptability to dynamic loads but higher costs and complexity are required. The recent advances in smart materials promoted the development of smart structures suitable for vibration damping and control. Between them the piezoelectric systems seem to be the most promising, however their efficiency relies on their placement. In a previous work the authors proposed and validated an analytical method to detect the optimal location of piezoelectric plates to control the multi-modal vibrations of a cantilever beam. Recent findings show that, if all actuators are activated simultaneously, the optimization problem can be traced back to the determination of the optimal potential distribution on all the piezoelectric actuators. In this paper the above method is taken into account and applied to a cantilever beam with 13 pairs of surface mounted PZT plates under the excitation provided by an electrodynamic shaker. The experimental damping of two flexural modes combinations has been performed by means of a special-purpose workbench and the assessment of the damping efficiency has been measured by means of a micro I.C.P. accelerometer. The results showed that the multimode vibrations of the cantilever beam can be efficiently damped if the potential distribution on all the PZT plates is optimized.
\end{abstract}

\section{Introduction}

The vibration control is one of the main challenges in many engineering fields. The structural vibrations may weaken the fatigue resistance and decrease the life-cycle of mechanical parts, so as to lead to the failure in the worst cases. In gas turbine engines, e.g., the vibrations arise from the interaction between the fluid and the blades [1,2], therefore many damping strategies have been proposed and implemented in order to increase the components integrity. In recent decades the smart materials have been studied for vibration control applications and, among them, the piezoelectric materials are considered the most promising because of their fast dynamic response. Several studies have shown that the efficiency of the piezoelectric elements can be enhanced by optimizing their placement on the structure [3, 4]. Previous studies

*e-mail: andrea.rossi@uniroma3.it 
from the authors [5-8] proposed an analytical model to optimize the piezoelectric elements placement to control the multimode vibrations of a cantilever beam. Subsequently this model has been further implemented in [9] with all the piezo actuators continuously activated and the optimization problem has been traced back to the identification of the optimal potential distribution on the elements that cover the beam. In this work the method has been experimentally tested by means of 13 pairs of surface mounted PZT plates and an electrodynamic shaker. The outcomes confirm that the multimode vibrations of a beam could be efficiently damped if the best potential distribution on all the PZT actuators is provided.

\section{Experimental setup}

In order to verify the proposed model an aluminum beam, covered with 13 pairs of PZT plates (Tab.1) was attached to a shaker by means of a purposely designed joint.

Table 1. Dimensions (in $\mathrm{mm}$ ) of the aluminium beam and PZT plates.

\begin{tabular}{lcccc}
\hline & Material & Lenght & Width & Thickness \\
\hline PZT actuator & PIC 255 (PI Ceramic) & 40 & 14 & 0.5 \\
Beam & Aluminium & 185 & 36 & 1.5 \\
\hline
\end{tabular}

The PZT plates and the shaker are supplied with a voltage composed of two harmonics (Eq.1) by two independent arbitrary function generators, so that two flexural modes can be elicited simultaneously:

$$
\mathbf{v}_{\text {piezo }}(t)=\mathrm{v}_{0}\left[(1-r) \cos \left(\omega_{\mathrm{i}_{1}} t\right)+r \cos \left(\omega_{\mathrm{i}_{2}} t\right)\right]
$$

where $\mathrm{v}_{0}$ ranges from 0 to $20 \mathrm{~V}, \omega_{\mathrm{i}_{1}}, \omega_{\mathrm{i}_{2}}$ are the modal frequencies considered, $r$ ranges from 0 to 1 and identifies the prevalence of a mode respect to the other. Referring to Fig. 1 the arbitrary function generator AFG1 provide $\mathrm{v}_{\text {piezo }}(t)$ (that can be magnified up to \pm 300 $\mathrm{V}_{\text {peak-to-peak }}$ ) to the PZT plates and AFG2, amplified by the power amplifier (SignalForce $G W-P A 300 E$ ) to the shaker. Every piezo plate is linked to a mechanical relay that can switch the sign of the $\mathrm{V}_{\text {piezo }}(t)$ by means of a microcontroller configured in a LabView environment. A data acquisition board (NI USB 6251) collect and elaborate the signal of a micro I.C.P. accelerometer ( $P C B$ 352A56) fixed on the free end of the beam. In such a way is possible to assess the damping efficacy of any potential distribution and compare them to verify if the maximum damping efficacy (i.e. the minimum displacement of the free end) is achieved when the theoretical optimal potential distribution is selected. The overall measurement setup uncertainty described before is estimated to be $5 \%$. 

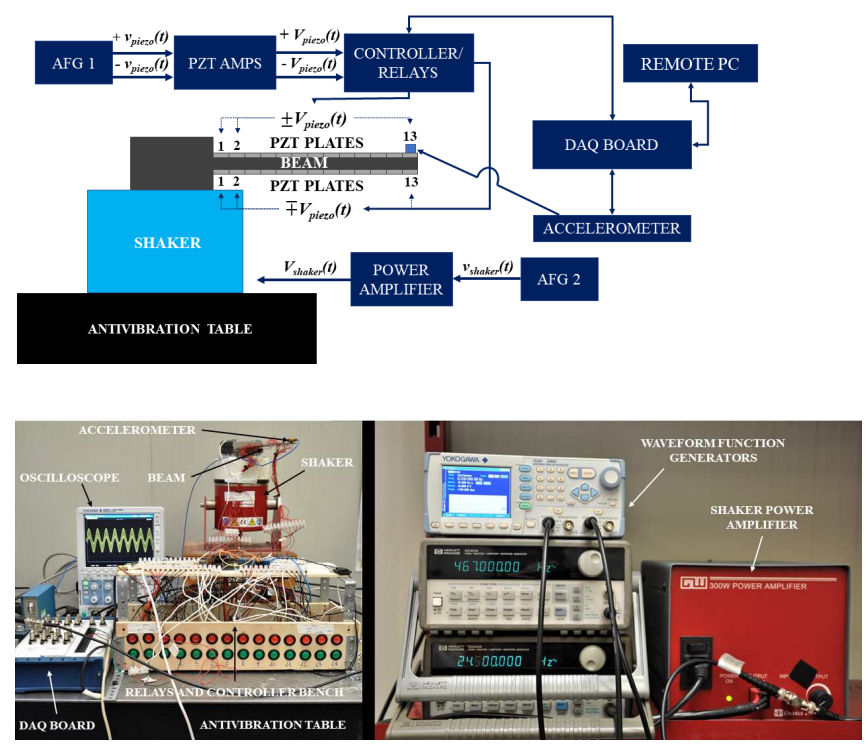

Figure 1. Experimental setup scheme (top) and actual setup photo (bottom).

\section{Results and discussion}

In this section the experimental results of the optimal potential distributions for multimode vibrations are reported and briefly commented. The dimensionless $\zeta_{1}, \zeta_{2}$ represent the points of the potential sign change (as illustrated in the example of Fig. 2). Since the beam is covered by 13 PZT plates couples, a large number of different potential distributions has been tested for each coupling modes.

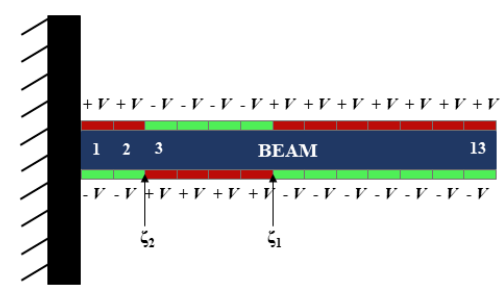

Figure 2. Example of a potential distribution acting on the PZT actuators.

In Figs 3-5 the experimental results are represented. Particularly, in each figure are reported the contour lines of the surfaces $\left|\mathrm{w}_{\mathrm{i}_{1}, \mathrm{i}_{2}}\left(\zeta_{1}, \zeta_{2}\right)\right|$ for different coupling modes $\mathrm{i}_{1}, \mathrm{i}_{2}$ and various values of the parameter $r$, and in the upper left the predictions of the theoretical model are illustrated. The details of the theoretical model are discussed in [9]. It is possible to observe that the experimental optimal potential distributions (the red region) are in good agreement with the theoretical results and all the potential sign changes are well reproduced. Furthermore the piezoelectric plates are always activated, regardless of the coupling modes elicited by the shaker. In such a way the damping efficiency of the PZT plates is enhanced. 

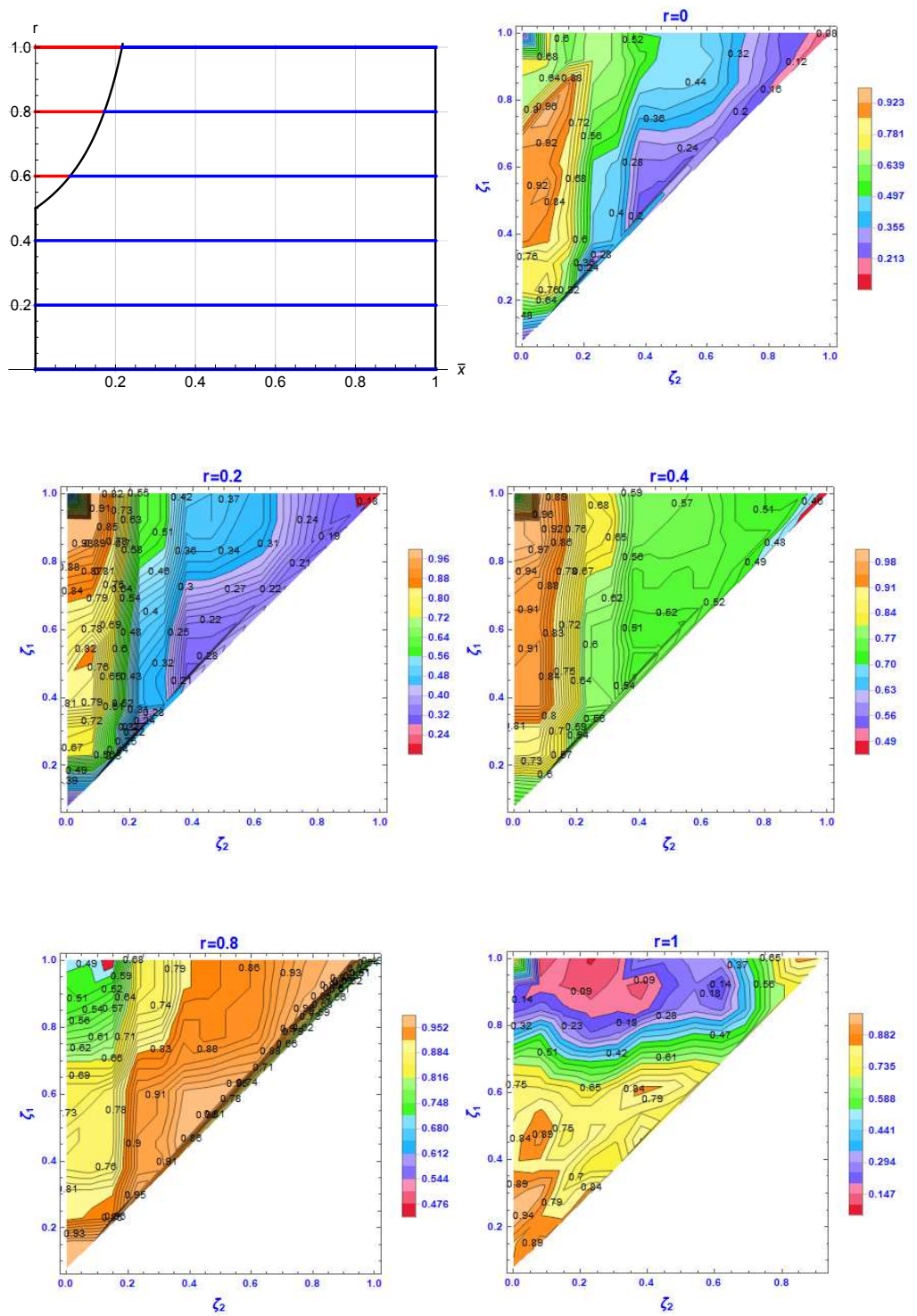

Figure 3. Experimental results for the coupling between the first and second modes for five values of $r$. The top left figure shows the theoretical optimal potential distributions. 

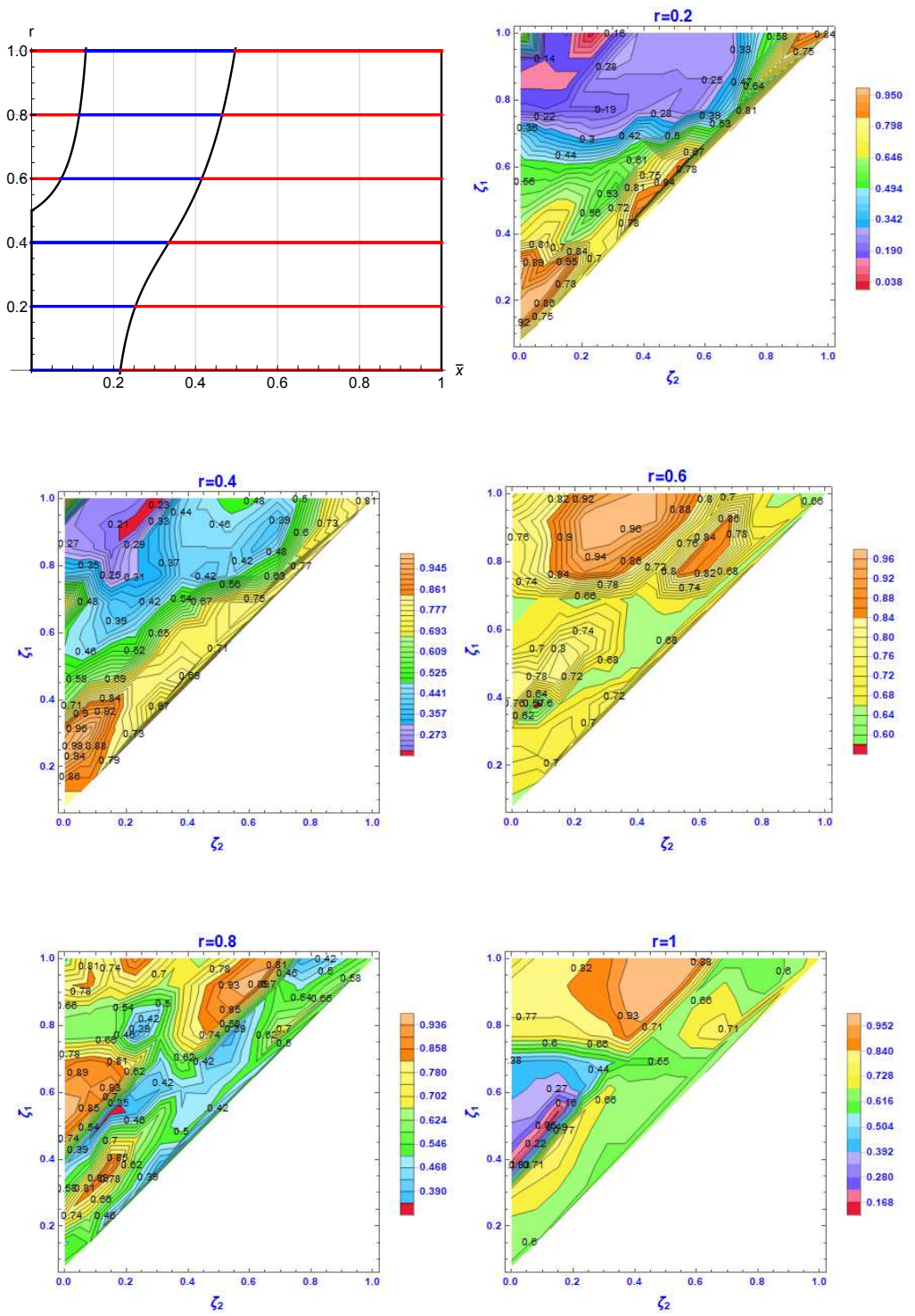

Figure 4. Experimental results for the coupling between the second and third modes for five values of $r$. The top left figure shows the theoretical optimal potential distributions. 

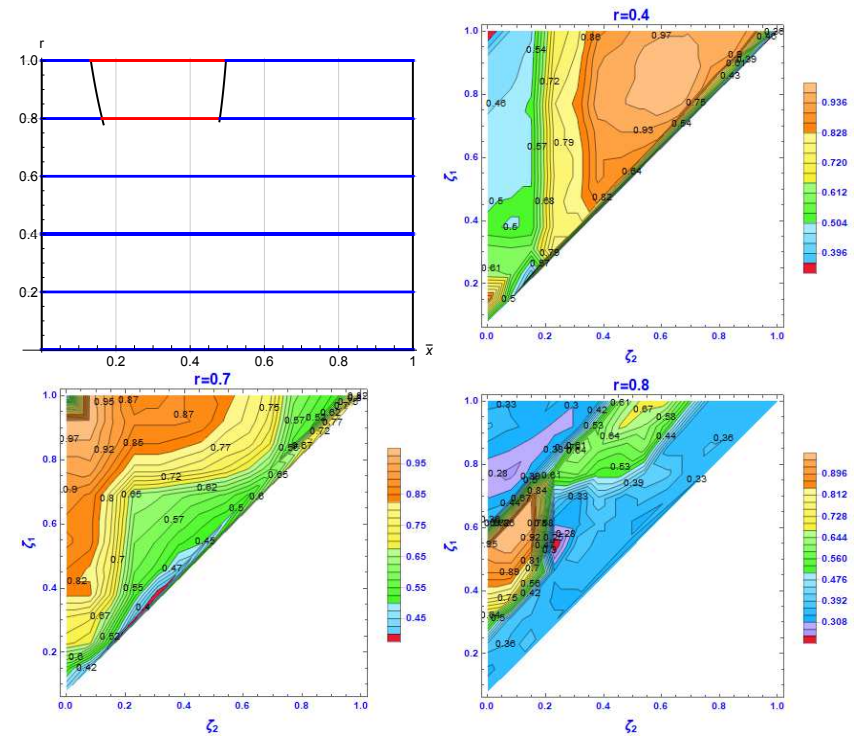

Figure 5. Experimental results for the coupling between the first and third modes for three values of $r$. The top left figure shows the theoretical optimal potential distributions.

\section{Conclusion}

In this work, the multimodal vibrations of an aluminium beam, induced by an electrodynamic shaker, were damped efficiently by means of 13 couples of PZT plates. The best damping action was found to be strictly related to the optimal distribution of the potential on the piezoelectric plates. Future works will focus on the development of a suitable experimental setup to verify the model extension in case of rotating beams, in order to assess the optimal actuators placement, e.g. for turbomachinery blade vibrations damping.

\section{References}

[1] J.Rao, Turbomachine blade vibration (New Age International, 1991)

[2] J.Rao, Turbomachine blade life estimation (New Age International, 2000)

[3] E.F. Crawley, J. De Luis, AIAA Journal, 25, 1373-1385 (1987)

[4] R. Barboni, A. Mannini, E. Fantini, P. Gaudenzi, Smart Materials and Structures, 9, 110 (2000)

[5] F. Botta, D. Dini, C. Schwingshackl, L. di Mare, G. Cerri, Advances in Acoustics and Vibration, 1, art. no. 905160 (2013)

[6] F. Botta, N. Marx, C. Schwingshackl, G. Cerri, D. Dini, Proceedings of the ASME Turbo Expo, 7A, Article number GT2013-95666 (2013)

[7] F. Botta, N. Marx, S. Gentili, C.W. Schwingshackl, L. Di Mare, G. Cerri, D. Dini, Sensors and Smart Structures Technologies for Civil, Mechanical, and Aerospace Systems, 8345, 180-184 (2012)

[8] F. Botta, A. Rossi, L. Schinaia, A. Scorza, F. Orsini, S.A. Sciuto, N.P. Belfiore, AIMETA 2017 Proceedings of the 23rd Conference of the Italian Association of Theoretical and Applied Mechanics, 3, 2258-2269 (2017)

[9] F. Botta, A. Scorza, A. Rossi, Applied Sciences, 8, 551 (2018) 